\title{
Bohmian Trajectories and the Path Integral Paradigm - Complexified Lagrangian Mechanics
}

\author{
Valery I. Sbitnev \\ B. P. Konstantionv St.-Petersburg Nuclear Physics Institute, \\ Russ. Ac. Sci., Gatchina, Leningrad District \\ Russia
}

\section{Introduction}

All material objects perceivable by our sensations move in real 3D-space. In order to describe such movement in strict mathematical forms we need to realize, first, what does the space represent as a mathematical abstraction and how motion in it can be expressed? Isaac Newton had gave many cogitations with regard to categories of the space and time. Results of these cogitations have been devoted to formulating categories of absolute and relative space and time (Stanford Encyclopeia, 2004): (a) material body occupies some place in the space; (b) absolute, true, and mathematical space remains similar and immovable without relation to anything external; (c) relative spaces are measures of absolute space defined with reference to some system of bodies or another, and thus a relative space may, and likely will, be in motion; (d) absolute motion is the translation of a body from one absolute place to another; relative motion is the translation from one relative place to another.

Observe, that space coordinates of a body can be attributed to center of mass of the body, and its velocity is measured as a velocity of motion of this center. It means, that a classical body can be replaced ideally by a mathematical point situated in the center of mass of the body. Velocity of the point particle is determined from movement of the center of mass per unit of time. Both point particle coordinates and its velocity are measured exactly. Its behavior can be computed unambiguously from formulas of classical mechanics (Lanczos 1970).

Appearance of quantum mechanics in the early twentieth century brought into our comprehension of reality qualitative revisions (Bohm, 1951). One problem, for example, arises at attempt of simultaneous measurement of the particle coordinate and its velocity. There is no method that could propose such measurements. Quantum mechanics proclaims weighty, nay, unanswerable principle of uncertainty prohibiting such simultaneous measurements. Therefore we can measure these parameters only with some accuracy limited by the uncertainty principle. From here it follows, that formulas of classical mechanics meet with failure as soon as we reach small scales. On these scales the particles behave like waves. It is said, in that case, about the wave-particle duality (Nikolić, 2007). 
It would be interesting to note here, that as far back as 5th century, B. C., ancient philosopher Democritus, (Stanford Encyclopeia, 2010), held that everything is composed of "atoms", which are physically indivisible smallest entities. Between atoms lies empty space. In such a view it means that the atoms move in the empty space. And only collisions of the atoms can effect on their future motions. One more standpoint on Nature, other than atomistic, originates from ancient philosopher Aristotle (Stanford Encyclopeia, 2008). Among his fifth elements (Fire, Earth, Air, Water, and Aether), composing the Nature, the last element, Aether, has a particular sense for explanation of wave processes. It provides a good basis for understanding and predicting the wave propagation through a medium.

By adopting wave processes underlying the Nature one can explain of interference phenomena of light. Huygens (Andresse, 2005) gave such an explanation. In contrast to Newtonian corpuscular explanation, Huygens proposed that every point to which a luminous wave reached becomes a source of a spherical wave, and the sum of these secondary waves determines the form of the wave at any subsequent time. His name was coined in the Huygens's wave principle, (Born \& Wolf, 1999).

Such a competition of the two standpoints, corpuscular and wave, can provide more insight penetration into problems taking place in the quantum realm. Here we adopt these standpoints as a program for action (Sbitnev, 2009a). The article consists of five sections. Sec. 2 begins from a short review of the classical mechanics methods and ends by Dirac's proposition as the classical action can show itself in the quantum realm. Feynman's path integral is a summit of this understanding. The path integral technique is used in Sec. 3 for computing interference pattern from $N$-slit gratings. In Sec. 4 the path integral is analyzed in depth. The Schrödinger equation results from this consideration. And as a result we get the Bohmian decomposition of the Schrödinger equation to pair of coupled equations, modified the Hamilton-Jacobi equation and the continuity equation. Sec. 5 studies this coupled pair in depth. And concluding Sec. 6 gives remarks relating to sensing our 3D-space on the quantum level.

\section{From classic realm to quantum}

A path along which a classical particle moves, Fig. 1, obeys to variational principles of mechanics. A main principle is the principle of least action (Lanczos, 1970). The action $S$ is a scalar function that is inner production of dynamical entities of the particle (its energy, momentum, etc.) to geometrical entities (time, length, etc.). For a particle's swarm moving through the space along some direction, the action is represented as a surface be pierced by their trajectories. Observe that adjoining surfaces are situated in parallel to each other and the trajectories pierce them perpendicularly.

The action $S$ is the time integral of an energy function, that is the Lagrange function, along the path from $A$ (starting from the moment $t_{0}$ ) to $B$ (finishing at the moment $t_{1}$ ):

$$
S=\int_{t_{0}}^{t_{1}} L(\vec{q}, \dot{\vec{q}} ; t) d t .
$$

Here $L(\vec{q}, \dot{\vec{q}} ; t)$ is the Lagrange function representing difference of kinetic and potential energies of the particle. And $\vec{q}$ and $\dot{\vec{q}}$ are its coordinate and velocity. Scientists proclaim that the action $S$ remains constant along an optimal path of the moving particle. It is the principle 


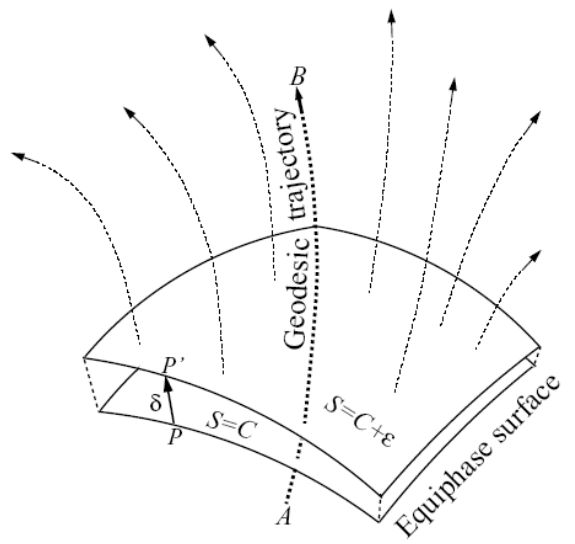

Fig. 1. Particle, at passing from $A$ to $B$, moves along geodesic trajectory - the trajectory satisfying the principle of least action. All geodesic trajectories intersect equiphase surfaces, $S=C, S=C+\varepsilon$, perpendicularly (Lanczos, 1970).

of least action. According to this principle, finding of the optimal path adds up to solution of the extremum problem $\delta S=0$. The solution leads to establishing the Lagrangian mechanics (Lanczos, 1970). We sum up the Lagrangian mechanics by presenting its main formulas via The Legendre's dual transformations as collected in Table 1:

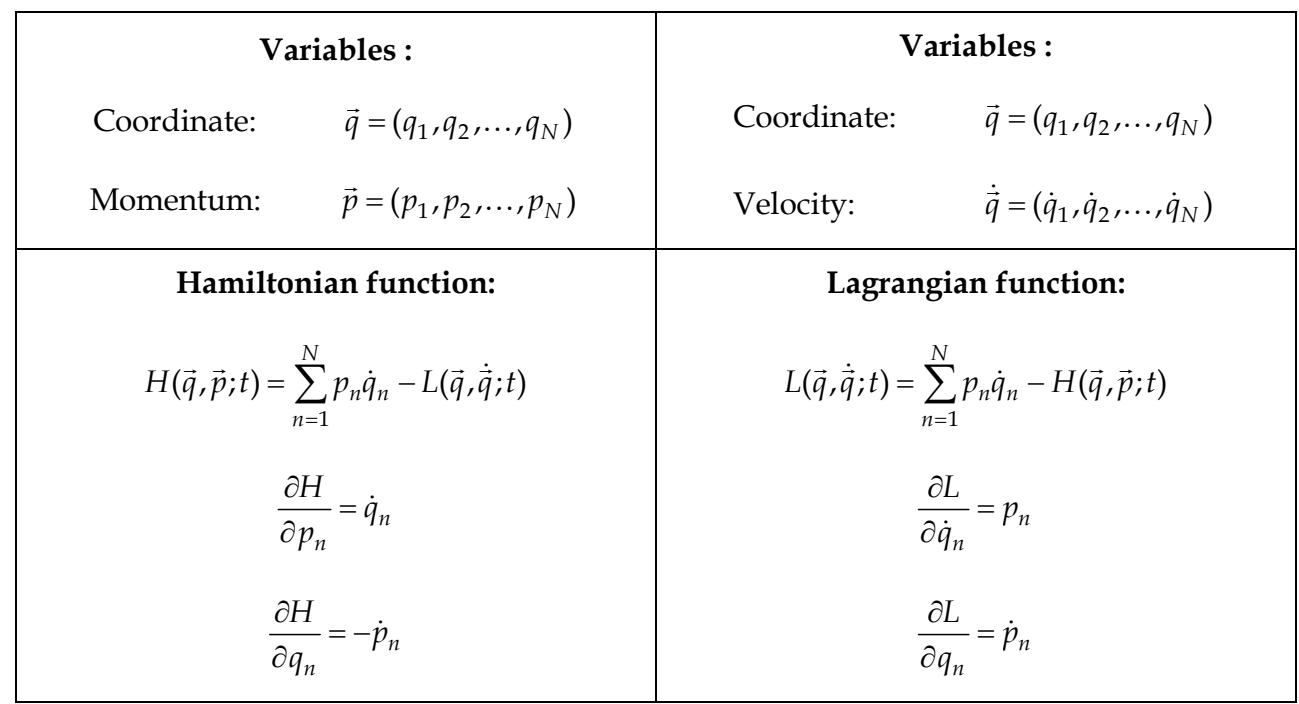

Table 1. The Legendre's dual transformations

The Hamilton-Jacobi equation (HJ-equation)

$$
-\frac{\partial S}{\partial t}=H(\vec{q}, \vec{p} ; t)
$$


describing behavior of the particle in $2 \mathrm{~N}$-dimesional phase space is one of main equations of the classical mechanics. Let us glance on Fig. 1. Gradient of the action $S$ can be seen as normal to the equiphase surface $S=$ const. Consider two nearby surfaces $S=C$ and $S=C+\varepsilon$. Let us trace normal from an arbitrary point $P$ of the first surface up to its intersection with the second surface at point $P^{\prime}$. Next, make another shift of the surface that is $2 \varepsilon$ distant away from the first surface, thereupon on $3 \varepsilon$, and so forth. Until all space will be filled with such secants. Normals drawn from $P$ to $P^{\prime}$ thereupon from $P^{\prime}$ to $P^{\prime \prime}$, and so forth, disclose possible trajectory of the particle, since $\nabla S=\varepsilon / \delta$ represents a value of the gradient of $S$. When $\varepsilon$ and $\delta$ tend to zero, this relation can be expressed in the vector form

$$
\vec{p}=\nabla S .
$$

So far as the momentum $\vec{p}=m \vec{v}$ ( $m$ is a particle mass) has a direction tangent to the trajectory, then the following statement is true (Lanczos, 1970): trajectory of a moving particle is perpendicular to the surface $S=$ const. Dotted curves in Fig. 1 show bundle of trajectories intersecting the surfaces $S$ perpendicularly.

The particle's swarm moving through space can be dense enough. It is appropriate to mention therefore the Liouville theorem, that adds to the conservation law of energy one more a conservation law. Meaning of the law is that a trajectory density is conserved independently of deformations of the surface that encloses these trajectories. Mathematically, this law is expressed in a form of the continuity equation

$$
\frac{\partial \rho}{\partial t}+(\vec{v} \cdot \nabla \rho)
$$

Here $\rho$ is a density of moving mechanical points with the velocity $\vec{v}=\vec{p} / m$.

Thus we have two equations, the HJ-equation (2) and the continuity equation (4) that give mathematical description of moving classical particles undergoing no noise. Draw attention here, that the continuity equation depends on solutions of the HJ-equation via the term $\vec{v}=\nabla S / m$. On the other hand we see, that the HJ-equation does not depend on solutions of the continuity equation. This is essential moment at description of moving ensemble of the classical objects.

Starting from a particular role of the action, which it has in classical mechanics, Paul Dirac drew attention in 1933 (Dirac, 1933) that the action can play a crucial role in quantum mechanics also. The action can exhibit itself in expressions of type $\exp \{$ iS $/ \hbar\}$. It is appropriate to notice the following observation: the action here plays a role of a phase shift. According to the principle of least action, we can guess that the phase shift should be least along an optimal path of the particle. In 1945 Paul Dirac emphasize once again, that the classical and quantum mechanics have many general points of crossing (Dirac, 1945). In particular, he had written in this article: "We can use the formal probability to set up a quantum picture rather close to the classical picture in which the coordinates $q$ of a dynamical system have definite values at any time. We take a number of times $t_{1}, t_{2}, t_{3}, \ldots$ following closely one after another and set up the formal probability for the $q$ 's at each of these times lying within specified small ranges, this being permissible since the $q$ 's at any time all commutate. We then get a formal probability for the trajectory of the system in quantum mechanics lying within certain limits. This enables us to speak of some trajectories being improbable and others being likely". 
Next, Richard Feynman undertook successful search of acceptable mathematical apparatus (Feynman, 1948) for description of evolution of quantum particles traveling through an experimental device. The term

$$
\exp \{\mathrm{i} S / \hbar\}=\exp \{\mathrm{i} L \delta t / \hbar\}
$$

plays a decisive role in this approach. Idea is that this term executes mapping of a wave function from one state to another spased on a small time interval $\delta t$. And $L$ is Lagrangian describing current state of the quantum object.

Feynman's insight has resulted in understanding that the integral kernel (so called propagator) of the time-evolution operator can be expressed as a sum over all possible paths (not just over the classical one) connecting the outgoing and ingoing points, $q_{a}$ and $q_{b}$, with the weight factor $\exp \left\{\mathrm{i} S\left(q_{a}, q_{b} ; t\right) / \hbar\right\}$ (Grosche, 1993; MacKenzie, 2000) :

$$
K\left(q_{a}, q_{b}\right)=\sum_{\text {all paths }} A \exp \left\{\mathrm{i} S\left(q_{a}, q_{b} ; T\right) / \hbar\right\},
$$

where $A$ is an normalization constant.

Observe that The Einstein-Smoluchowski equation which describes the Brownian motion of classical particles within some volume (Kac, 1957), served him as an example. As follows from idea of the path integral (6), there are many possible trajectories, that can be traced from a source to a detector. But only one trajectory, submitting to the principle of least action, may be real. The others cancel each other because of interference effects. Such an interpretation is extremely productive at generating intuitive imagination for more perfect understanding quantum mechanics.

It is instructive further to consider some quantum tasks by using the Feynman path integral. Here we will compute interference patterns as a result of incidence of particles on $\mathrm{N}$-slit gratings.

\section{Interference pattern from an $\mathbf{N}$-slit gratin}

Let a beam of coherent particles spreads through a grating. The grating shown in Fig. 2 has a set of narrow slits sliced in parallel. Width of the slits is sufficient in order that even large molecules could pass they through. Here we face with the uncertainty principle, $\Delta r \Delta p \geq \hbar / 2$.

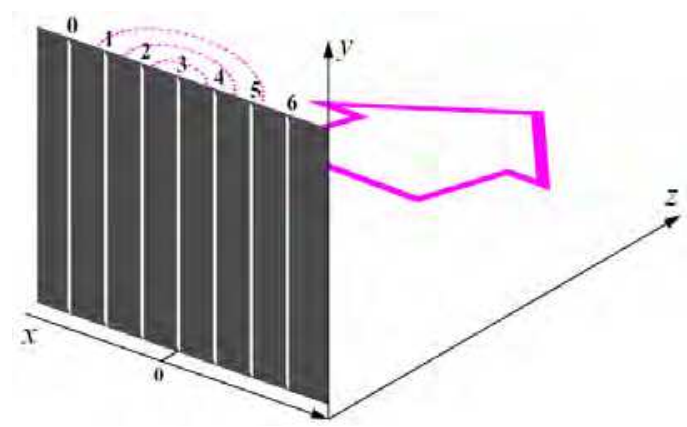

Fig. 2. Interference experiment in cylindrical geometry. Slit grating with $n=0,1, \ldots, N-1$ slits is situated in a plane $(x, y)$. Propagation of particles occurs along axis $z$. 
It means, if diameter of the molecule is close to width of the slit then direction of its escape from the slit is uncertain. One can draw, as commonly, cylindrical waves that are divergent from each slit, as shown in this illustration on the slit 3. They illustrate equally probable outcomes from slits in different directions. In other words, a particle may fly out in any direction with equal probability.

\subsection{Passing through a slit}

Before we will analyze interference on the $N$-slit grating, let us consider a particle passing through a single slit. The problem has been considered in detail in (Feynman \& Hibbs, 1965). We will study migration of the free particle in transversal direction, let it be axis $x$, at passing along $z$ with a constant velocity, see Fig. 3. Lagrangian is as follows

$$
L=m \frac{\dot{x}^{2}}{2}+\text { const }
$$

Here $m$ is mass of the particle and $\dot{x}$ is its transversal velocity. By translating a particle's position on a small value $\delta x=\left(x_{b}-x_{a}\right)<<1$, being performed for a small time $\delta t=\left(t_{b}-t_{a}\right)<<1$, we find that a weight factor, see (5), is as follows

$$
\mathrm{e}^{\mathrm{i} L \delta t / \hbar}=\exp \left\{\frac{\mathrm{i} m\left(x_{b}-x_{a}\right)^{2}}{2 \hbar\left(t_{b}-t_{a}\right)}\right\} .
$$

Pay attention on the following situation: so far as argument of the exponent contains multiplication of the Lagrangian $L$ by $\delta t$, as shown in Eq. (5), we obtain result $\left(x_{b}-x_{a}\right)^{2}$ divided by $\left(t_{b}-t_{a}\right)$. Next we will see, that the weight factor (8) plays an important role. By means of such small increments let us trace passing the particle from a source through the slit, Fig. 3.

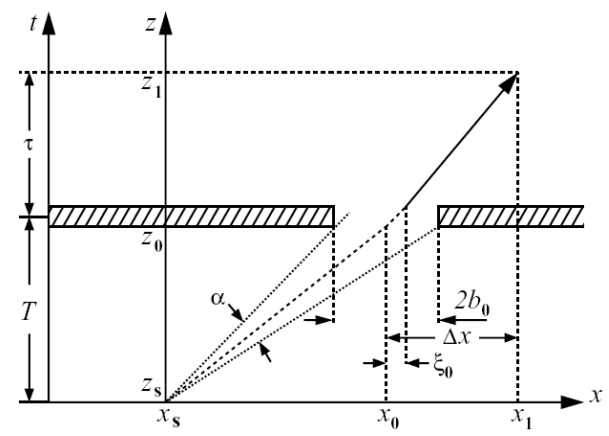

Fig. 3. A particle, being emitted from a source that is localized at a point $\left(x_{\mathrm{s}}, z_{\mathrm{s}}\right)$ passes through a slit with width $2 b_{0}$. It may undergo deflection from a straight direction at passing through the slit (Feynman \& Hibbs, 1965).

We suppose, that at the time $t=0$ the particle leaves a source localized at a point $\left(x_{\mathrm{s}}, z_{\mathrm{s}}\right)$. Let we know, that after a time $T$ the particle enters to the vicinity $x_{0} \pm b$ of a point $x_{0}$, see Fig. 3 . The question is: what is the probability to disclose the particle after a time $\tau$ at a point $x_{1}$ remote from the point $\mathrm{x}_{0}$ at a distance $\Delta x=\left(x_{1}-x_{0}\right)$ ? Let the particle outgoing from the point 
$x_{\mathrm{s}}$ at the time $t=0$ passes a slit between the points $x_{0}-b$ and $x_{0}+b$ at the time $t=T$. Let us compute the probability of discovering the particle at some point $\mathrm{x}_{1}$ after the time $\tau$, i.e., at $t=T+\tau$. Because of existence of an opaque barrier a direct path to the point $x_{1}$ can be absent. In order to reach the point $x_{1}$ the particle should pass through the slit, maybe with some deflection from the direct path. In this connection, we partition the problem into two parts. Each part relates to movement of the free particle. In the first part we consider the particle which begins movement from the point $x_{\mathrm{s}}$ at the initial moment $t=0$ and reaches to a point $x$ $=x_{0}+\xi$, at the moment $t=T$, where $|\xi| \leq b$. In the second part we consider the same particle that after passing the point $x=x_{0}+\xi$ at the time $t=T$ moves to the point $x_{1}$ and reach it at the time $t=T+\tau$. A full probability amplitude is equal to integral convolution of two kernels, each describing movement of the free particle:

$$
\psi\left(x_{1}, x_{0}, x_{\mathrm{s}}\right)=\int_{-b}^{b} K\left(x_{1}, T+\tau ; x_{0}+\xi, T\right) K\left(x_{0}+\xi, T ; x_{\mathrm{s}}, 0\right) d \xi .
$$

Here the kernel reads

$$
K\left(x_{b}, t_{b} ; x_{a}, t_{a}\right)=\left[\frac{2 \pi \mathrm{i} \hbar\left(t_{b}-t_{a}\right)}{\mathrm{m}}\right]^{-1 / 2} \exp \left\{\frac{\mathrm{i} m\left(x_{b}-x_{a}\right)^{2}}{2 \hbar\left(t_{b}-t_{a}\right)}\right\} .
$$

It describes a transition amplitude from $x_{a}$ to $x_{b}$ for a time interval $\left(t_{b}-t_{a}\right)$ (Feynman \& Hibbs, 1965). Consequently, the integral (9) computes the probability amplitude of transition from the source $x_{\mathrm{s}}$ to the point $\mathrm{x}_{1}$ through the all possible intermediate points $\xi$ situated within the interval $\left(x_{0}-b, x_{0}+b\right)$.

The expression (9) is written in accordance with a rule of summing amplitudes for successive events in time. The first event is the moving particle from the source to the slit. The second event is the movement of the particle from the slit to the point $x_{1}$. The slit has a finite width. Passage through the slit is conditioned by different alternative possibilities. For that reason, we need to integrate along all over the slit width in order to get a right result. All particles, moving through the slit, are free particles and their corresponding kernels are given by the expression (10). By substituting this kernel to the integral (9) we get the following detailed form

$$
\psi\left(x_{1}, x_{0}, x_{\mathrm{s}}\right)=\int_{-b}^{b}\left(\frac{2 \pi \mathrm{i} \hbar \tau}{m}\right)^{-1 / 2} \exp \left\{\frac{\mathrm{i} m(\Delta x-\xi)^{2}}{2 \hbar \tau}\right\}\left(\frac{2 \pi \mathrm{i} \hbar T}{m}\right)^{-1 / 2} \exp \left\{\frac{\mathrm{i} m\left(x_{0}-x_{\mathrm{s}}+\xi\right)^{2}}{2 \hbar T}\right\} d \xi .
$$

Integration here is fulfilled along the slit of a width $a=2 b$, i.e., from $-b$ to $+b$.

Formally, range of the integration can be broadened from $-\infty$ to $+\infty$. But in this case, we need to introduce the step function $G(\zeta)$ equal to unit in the interval $[-b,+b]$ and equal to zero outside this interval. In principle, we can approximate hard edged slits by series of the Gaussian functions, each with narrow halfwidth (Sbitnev, 2010). For sake of simplicity however, we confine themselves by a single Gaussian form-factor

$$
G(\xi)=\exp \left\{-\xi^{2} / 2 b^{2}\right\} .
$$

It simulates slits with fuzzy edges. Effective width of this curve is conditioned by a parameter $b$. For such a form-factor roughly two thirds of all its area is situated between the 
points $-b$ and $+b$. If the particles would move by classical way, then we can anticipate, that after the time $\tau$ a distribution of the particles will be similar to the distribution existing at $T$, see Fig. 4 . New center $x_{1}$ of the distribution is shifted on a value $\Delta x$ from the point $x_{0}$. Width $b_{1}$ of the new distribution is also broadened. The both parameters, $x_{1}$ and $b_{1}$, are determined from expressions

$$
x_{1}=x_{0}\left(1+\frac{\tau}{T}\right), \quad b_{1}=b\left(1+\frac{\tau}{T}\right)
$$

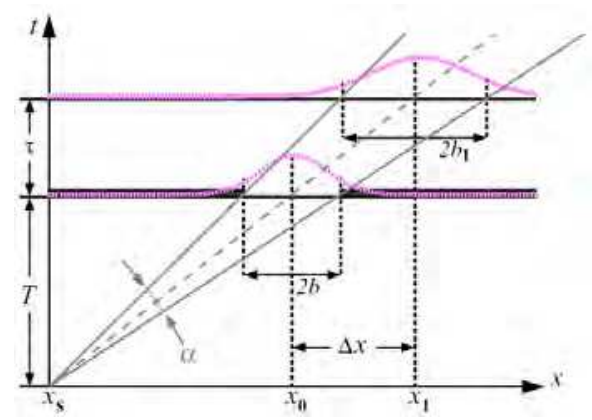

Fig. 4. Trajectories of particles passing through the Gaussian slit (Feynman \& Hibbs, 1965), form a ray with an angle $\alpha$ of the divergent particle beam emanating from the source $x_{\mathrm{s}}$.

Observe that quantum particles, in contrast to the classical ones, at scattering on the slit behave themselves like waves. The wavelike nature manifests itself via phase shifts of the moving particles in an observation point because of the de Broglie wavelength as innate character of quanta. According to the above stated remarks, Eq. (11) with inserted the formfactor of the slit, $G(\zeta)$, now can be rewritten in the following form

$$
\psi\left(x_{1}, x_{0}, x_{\mathrm{s}}\right)=\int_{-\infty}^{\infty} \frac{m G(\xi)}{2 \pi \mathrm{i} \hbar \sqrt{T \tau}} \cdot \exp \left\{\frac{\mathrm{i} m}{2 \hbar}\left[\frac{\left(x_{1}-x_{0}-\xi\right)^{2}}{\tau}+\frac{\left(x_{0}-x_{\mathrm{s}}+\xi\right)^{2}}{T}\right]\right\} d \xi .
$$

By substituting $G(\xi)$ from Eq. (12) to this expression and integrating it we obtain

$$
\begin{gathered}
\psi\left(x_{1}, x_{0}, x_{\mathrm{s}}\right)=\sqrt{\frac{m}{2 \pi \mathrm{i} \hbar T}}\left(1+\frac{\tau}{T}+\mathrm{i} \frac{\hbar \tau}{m b^{2}}\right)^{-1 / 2} \\
\exp \left\{\frac{\mathrm{i} m}{2 \hbar}\left(\frac{\left(x_{1}-x_{0}\right)^{2}}{\tau}+\frac{\left(x_{0}-x_{\mathrm{s}}\right)^{2}}{T}-\frac{\left(\left(x_{1}-x_{0}\right) / \tau-\left(x_{0}-x_{\mathrm{s}}\right) / T\right)^{2} \tau}{\left(1+\tau / T+\mathrm{i} \hbar \tau / m b^{2}\right)}\right)\right\}
\end{gathered}
$$

At integrating Eq. (14) we use a standard integral

$$
\int_{-\infty}^{\infty} e^{\alpha \xi^{2}+\beta \xi+\gamma} d \xi=\sqrt{\frac{\pi}{-\alpha}} e^{-\beta^{2} / 4 \alpha+\gamma} .
$$

Before we will write out a final expression let us fulfill a series of replacements. 


\subsubsection{Series of replacements}

First we define an effective slit's half-width $\sigma_{0}=b / \sqrt{2}$. And further we define a complex time-dependent spreading

$$
\sigma_{\tau}=\sigma_{0}+\mathrm{i} \frac{\hbar \tau}{2 m \sigma_{0}(1+\tau / T)}
$$

which has been defined in works (Sanz \& Miret-Artês, 2007, 2008\}. More one step is to replace flight times $T$ and $\tau$ by flight distances $\left(z_{0}-z_{\mathrm{s}}\right)$ and $\left(z_{1}-z_{0}\right)$, namely, $T=\left(z_{0}-z_{\mathrm{s}}\right) / v_{\mathrm{z}}$ and $\tau=\left(z_{1}-z_{0}\right) / v_{z}$. Here $v_{z}$ is a particle velocity along the axis $z$. We note that $m v_{z}=p_{z}$ is $z-$ component of the particle momentum. This component is not changed at passing through the grating. Next, we introduce the de Broglie wavelength $\lambda_{\mathrm{dB}}=h / p_{\mathrm{z}}$, where $h=2 \pi \hbar$ is the Planck constant. Rewrite in this view the complex time-dependent spreading (17) as the complex distance-dependent spreading

$$
\sigma_{\tau \rightarrow z_{1}}=\sigma_{0}+\mathrm{i} \frac{\lambda_{\mathrm{dB}}\left(z_{1}-z_{0}\right)}{4 \pi \sigma_{0}\left(\frac{z_{1}-z_{s}}{z_{0}-z_{s}}\right)}
$$

Define now a dimensionless complex distance-dependent spreading as follows

$$
\Sigma_{z_{1}}=\frac{z_{1}-z_{s}}{z_{0}-z_{s}}+\mathrm{i} \frac{\lambda_{\mathrm{dB}}\left(z_{1}-z_{0}\right)}{4 \pi \sigma_{0}^{2}}
$$

and a dimensionless parameter characterizing remoteness of the source

$$
\Xi_{0}=1-\frac{\left(x_{0}-x_{s}\right)}{\left(z_{0}-z_{s}\right)} \frac{\left(z_{1}-z_{0}\right)}{\left(x_{1}-x_{0}\right)}
$$

which tends to 1 as $z_{s} \rightarrow-\infty$.

Now we can use the above parameters, the dimensionless complex distance-dependent spreading $\Sigma_{z_{1}}$ and the remoteness of the source $\Xi_{0}$, in order to write out the wave function behind the slit. By rewriting Eq. (15) via these parameters we obtain

$$
\psi\left(x, x_{0}, x_{s}, z\right)=\sqrt{\frac{m}{2 \pi \mathrm{i} \hbar T \Sigma_{z}}} \exp \left\{\mathrm{i} \pi\left[\frac{\left(x-x_{0}\right)^{2}}{\lambda_{\mathrm{dB}}\left(z-z_{0}\right)}\left(1-\frac{\Xi_{0}^{2}}{\Sigma_{z}}\right)+\frac{\left(x_{0}-x_{s}\right)^{2}}{\lambda_{\mathrm{dB}}\left(z_{0}-z_{s}\right)}\right]\right\} .
$$

Here we have removed the subscript 1 at the variables, $x, z$, and $\Sigma_{z}$, since they relate to every points of the space behind the slit. In particular, at removing the source to infinity, $z_{\mathrm{s}} \rightarrow-\infty$, the parameter $\Xi_{0}$ tends to 1 and the wave function reduces down to the paraxial approximation

$$
\psi\left(x, x_{0}, z\right)=A \exp \left\{\mathrm{i} \pi \frac{\left(x-x_{0}\right)^{2}}{\lambda_{\mathrm{dB}}\left(z-z_{0}\right)}\left(1-\frac{1}{\Sigma_{z}}\right)\right\} .
$$

Here a normalization factor $A$ reads 


$$
A=\sqrt{\frac{m}{2 \pi \mathrm{i} \hbar T \Sigma_{z}}} .
$$

One can see it vanishes at $T \rightarrow \infty$. It means, as the source moves away to infinity its intensity tends to zero. In the paraxial approximation we need to ignore this expression and consider the parameter $A$ simply as a factor that normalizes the wave function.

Further, for the sake of simplicity, we will deal with the paraxial approximation.

\subsection{Matter waves behind the grating}

Let we have a screen, on which an incident monochromatic beam of the particles is scattered. It has $N$ slits $(n=0,1,2, \ldots, N-1)$ located at equal distance from each other, as shown in Fig. 2. Origin of coordinates is placed in the center of the slit grating. In this frame of reference, $n$-th slit has a position $x_{0}=(n-(N-1) / 2) d$, where $d$ is a spacing between slits. The spacing is measured in units multiple to the wavelength $\lambda_{\mathrm{dB}}$.

We need now to compute contributions of all paths that pass from the source through all slits in the screen and farther to a point of observation $(x, z)$. Per se, we should superpose in the observation point all wave functions (22) from all slits $n=0,1,2, \ldots, N-1$. Such a superposition reads

$$
|\Psi(x, z)\rangle=\frac{1}{N} \sum_{n=0}^{N-1} \psi\left(x,\left(n-\frac{N-1}{2}\right) d, z\right)
$$

and probability density in the vicinity of the observation point $(x, z)$ is

$$
p(x, z)=\langle\Psi(x, z) \mid \Psi(x, z)\rangle .
$$

\subsubsection{Far-field diffraction}

Before we will take up interference effects in the near-field region, let us consider an asymptotic limit of the formula (25) in the far-field region, Fig. 5. With this aim in mind, we replace the term $(n-(N-1) / 2) d$ in Eq. (24) by $k d$, where $k$ runs from $-(N-1) / 2$ to $(N-1) / 2$. Next, at summation we will neglect contribution of coefficients at $k^{2} d{ }^{2}$ emergent at decomposition $(x-k d)^{2}=x^{2}-2 x k d+k^{2} d^{2}$. The point is that the terms with $k^{2} d^{2}$ lead to phases muddled up on infinity. Because of it sum of all these exponents gives zero contribution. Other sums containing coefficients at $x^{2}$ and $2 x k d$ can be easily computed. Next, at summation we use the mathematical equality

$$
\sum_{k=-(N-1) / 2}^{(N-1) / 2} \exp \{\mathrm{i} k x\}=\frac{\sin (N x / 2)}{\sin (x / 2)} .
$$

Intensity of the particle beam in the far-field region computed according to the above approximation is as follows

$$
I(x, z)=I_{0}(x, z) \frac{\sin \left(\frac{N \zeta(x, z)}{2}\right)^{2}}{\sin \left(\frac{\zeta(x, z)}{2}\right)^{2}}
$$




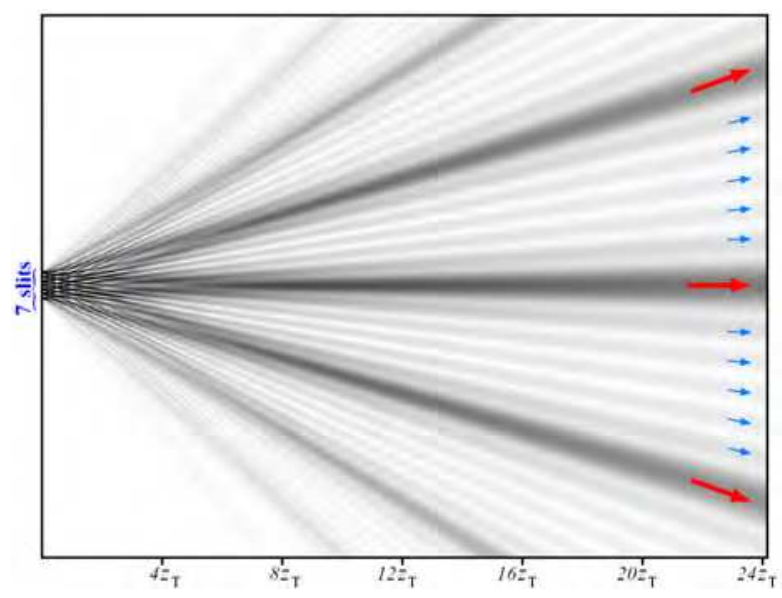

Fig. 5. Diffraction in the far-field zone at simulation of scattering thermal neutrons $\left(\lambda_{\mathrm{dB}}=0.5\right.$ $\mathrm{nm}$ ) on $N=7$ slits grating. Width of slits $a=2 \lambda_{\mathrm{dB}}$, spacing $d=10 \lambda_{\mathrm{dB}}$, and the Talbot length $z_{\mathrm{T}}=2 d^{2} / \lambda_{\mathrm{dB}}=200 \lambda_{\mathrm{dB}}$. Directions of principal and subsidiary maxima are pointed out by big red arrows and small blue arrows, respectively.

Here terms $\zeta(x, z)$ and $I_{0}(x, z)$ read

$$
\left\{\begin{array}{c}
\zeta(x, z)=\frac{x d \frac{z \lambda_{\mathrm{dB}}}{4 \pi \sigma^{2}}}{2 \sigma_{\mathrm{z}}^{2}}, \\
\mathrm{I}_{0}(x, z)=\frac{A^{2}}{N^{2} \sigma_{z}} \exp \left\{-\frac{x^{2}}{2 \sigma_{\mathrm{z}}^{2}}\right\} .
\end{array}\right.
$$

The parameter $A$ is the normalization factor, see Eq. (23), and $\sigma_{\mathrm{z}}$ has the following form

$$
\sigma_{z}=\sigma \sqrt{1+\left(\frac{z \lambda_{\mathrm{dB}}}{4 \pi \sigma^{2}}\right)} .
$$

This parameter is equivalent to the instantaneous Gaussian width presented in (Sanz and Miret-Artês, 2007).

Fig. 6 shows diffraction in the far-field zone from the grating having $N=7$ slits. Distance to the observation screen is $z=10^{7} z_{\mathrm{T}}=1 \mathrm{~m}$, where $z_{\mathrm{T}}=2 d^{2} / \lambda_{\mathrm{dB}}=200 \lambda_{\mathrm{dB}}$ is the Talbot length. It will be explained below. It is seen, that the principal maxima are partitioned from each other by $N-2=5$ subsidiary maxima.

\subsubsection{Near-field interference}

Above we have considered a coherent flow of thermal neutrons, $\lambda_{\mathrm{dB}}=0.5 \mathrm{~nm}$. Radius of these particles is $10^{-15} \mathrm{~m}$. It is much smaller the de Broglie wavelength $\lambda_{\mathrm{dB}}=5 \cdot 10^{-10} \mathrm{~m}$. For this reason, these particles can be considered as point particles, in contrast to enormous fullerene molecules shown in Fig 7. 


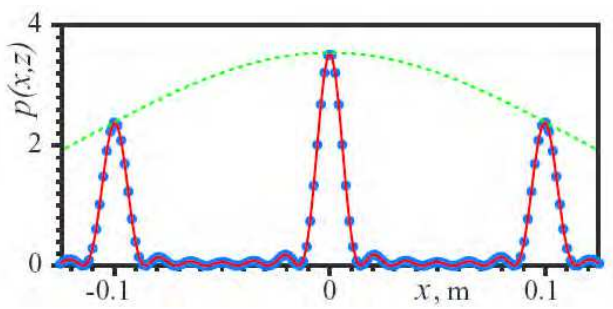

Fig. 6. Diffraction of thermal neutrons $\left(\lambda_{\mathrm{dB}}=0.5 \mathrm{~nm}\right)$ in the far-field zone from grating having $N=7$ slits. Distance to observation screen is $z=1 \mathrm{~m}$. Blue circles relate to the probability density calculated by Eqs. (24)--(25). Intensity (27) is drawn by red solid curve. Dotted green curve draws envelope $I_{0}(x, z) N^{2}$.

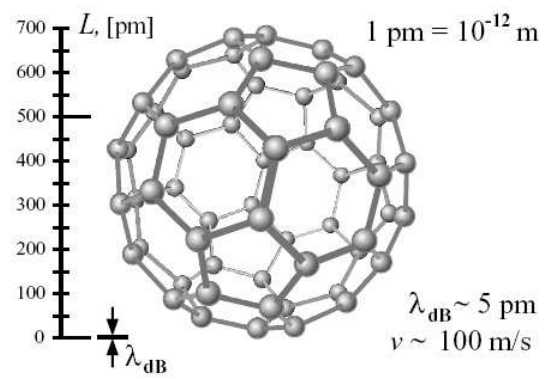

Fig. 7. The fullerene molecule $\mathrm{C}_{60}$ consists of 60 carbon atoms. Its radius is about $700 \mathrm{pm}$. At a flight velocity from a source $v=100 \mathrm{~m} / \mathrm{s}$ de Broglie wavelength of the fullerene molecule, $\lambda_{\mathrm{dB}}$, is about $5 \mathrm{pm}$.

Here we consider interference phenomena in the near-field created by the fullerene molecules. Interest to such heavy molecules, having masses about $100 \mathrm{amu}$ and more (Arndt et al., 2005; Brezger et al., 2002, 2005; Gerlich et al., 2011; Hackermüller et al., 2003, 2004; Nairz et al., 2003) is due to the fact that under ordinary circumstances they behave almost as classical objects. Indeed, diameter of the fullerene molecule $\mathrm{C}_{60}$, see Fig. 7, is about $700 \mathrm{pm}$ (Yanov \& Leszczynski, 2004), but de Broglie wavelength is 5 pm (Hackermüller et al., 2003; Juffmann et al., 2009). There is a problem to observe quantum interference for such large molecules having minuscule wavelengths.

At small distances from the grating we need in a acceptable scale in order to partition interference patterns on characteristic zones. Such a scale parameter is the Talbot length

$$
z_{\mathrm{T}}=2 \frac{d^{2}}{\lambda_{\mathrm{dB}}} .
$$

This length starts from Henry Fox Talbot who discovered in 1836 a beautiful interference pattern (Talbot, 1836), that carries his name. Here $d$ is the spacing between slits and $\lambda_{\mathrm{dB}}$ is the de Broglie wavelength of particles under consideration. Figs. 8 and 9 show emergence of such interference patterns in the near-field.

Fig. 8 shows the density distribution function (25) in a transient region from near-field to far-field (it is shown in gray color). The Talbot length ranges from 0 to $8 z_{\mathrm{T}}=0.8 \mathrm{~m}$. The 


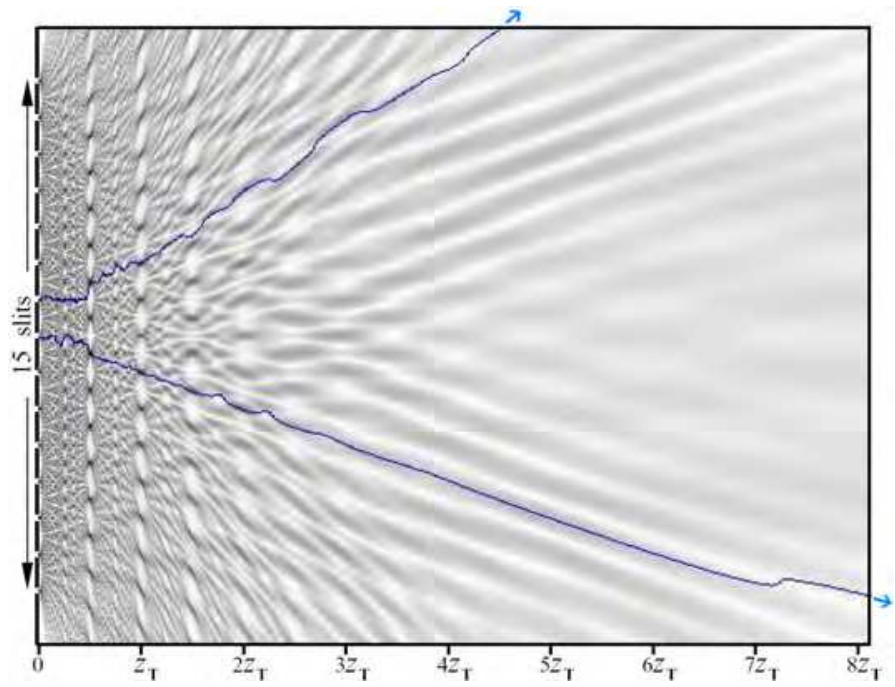

Fig. 8. Interference pattern of matter waves. The wave is presented by coherent fullerene molecule beam incident to a grating having $N=15$ slits. De Broglie wavelength of the fullerene molecules is $\lambda_{\mathrm{dB}}=5 \mathrm{pm}$. Spacing between slits $d=500 \mathrm{~nm}$ and slit width $a=2 b=10$ $\mathrm{nm}$. The Talbot length $z_{\mathrm{T}}=0.1 \mathrm{~m}$. Two Bohmian trajectories divergent from central area of the grating are shown in blue as examples.

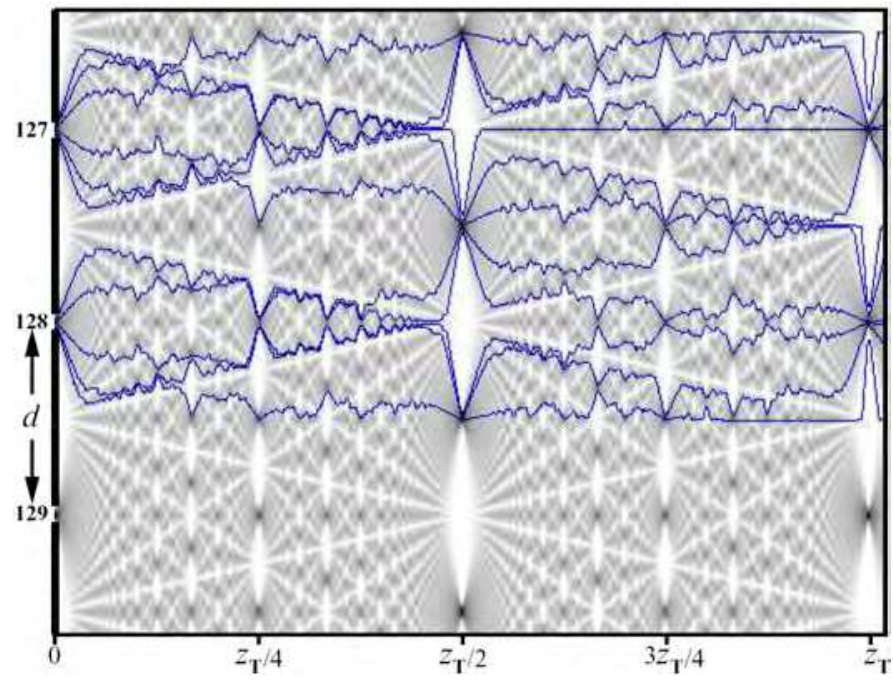

Fig. 9. Talbot carpet in the near-field of the grating having $N=255$ slits. The pattern has been captured from central part of the grating. De Broglie wavelength of the fullerene molecules is $\lambda_{\mathrm{dB}}=5 \mathrm{pm}$. Spacing between slits $d=500 \mathrm{~nm}$ and slit width $a=2 b=10 \mathrm{~nm}$. The Talbot length $z_{\mathrm{T}}=0.1 \mathrm{~m}$. Some of the Bohmian trajectories passing by zigzag through spots with high density distribution are shown in blue as examples. 
interference pattern emergent has been calculated for heavy particles, fullerene molecules, Fig. 7, incident on the grating containing $N=15$ slits. Spacing between slits is $d=500 \mathrm{~nm}$ and slit width $a=2 b=10 \mathrm{~nm}$. Mass of the fullerene molecules is about $\mathrm{m}_{\mathrm{C} 60} \approx 1 \cdot 2 \cdot 10^{-24} \mathrm{~kg}$. And at average velocity about $100 \mathrm{~m} / \mathrm{s}$ (Juffmann et al., 2009) the de Broglie wavelength is 5 $\mathrm{pm}$. The Talbot length is about $\mathrm{z}_{\mathrm{T}}=0.1 \mathrm{~m}$. One can easily evaluate that ratio of the Talbot length to the spacing between slits is equal to $2 \cdot 10^{5}$. So, a stripe between two slits extending from the grating up to the first Talbot length is extremely narrow. We can see that nearby the grating there exists a relatively perfect interference pattern. It decays with removing from the grating. And far from the grating characteristic rays divergent from it arise, as shown, for example, in Fig. 5

More fascinating picture arises at observation of the Talbot carpet as a peculiar manifestation of interference in near-field, see Fig. 9. The Talbot carpets arise if three conditions, Berry's conditions (Berry, 1996, 1997; Berry \& Klein, 1996; Berry et al., 2001), are fulfilled: (a) paraxial beam; (b) arbitrary small ratio $\lambda_{\mathrm{dB}} / d$; (c) arbitrary large number of slits. In a strict sense, in the limits $N \rightarrow \infty$ and $\lambda_{\mathrm{dB}} / d \rightarrow 0$ the Talbot carpet should transform to fractal interference pattern. It would look like $\delta$-peaks everywhere densely populating the probability density distribution function $p(x, z)$, as shown, for example, in Fig. 10.

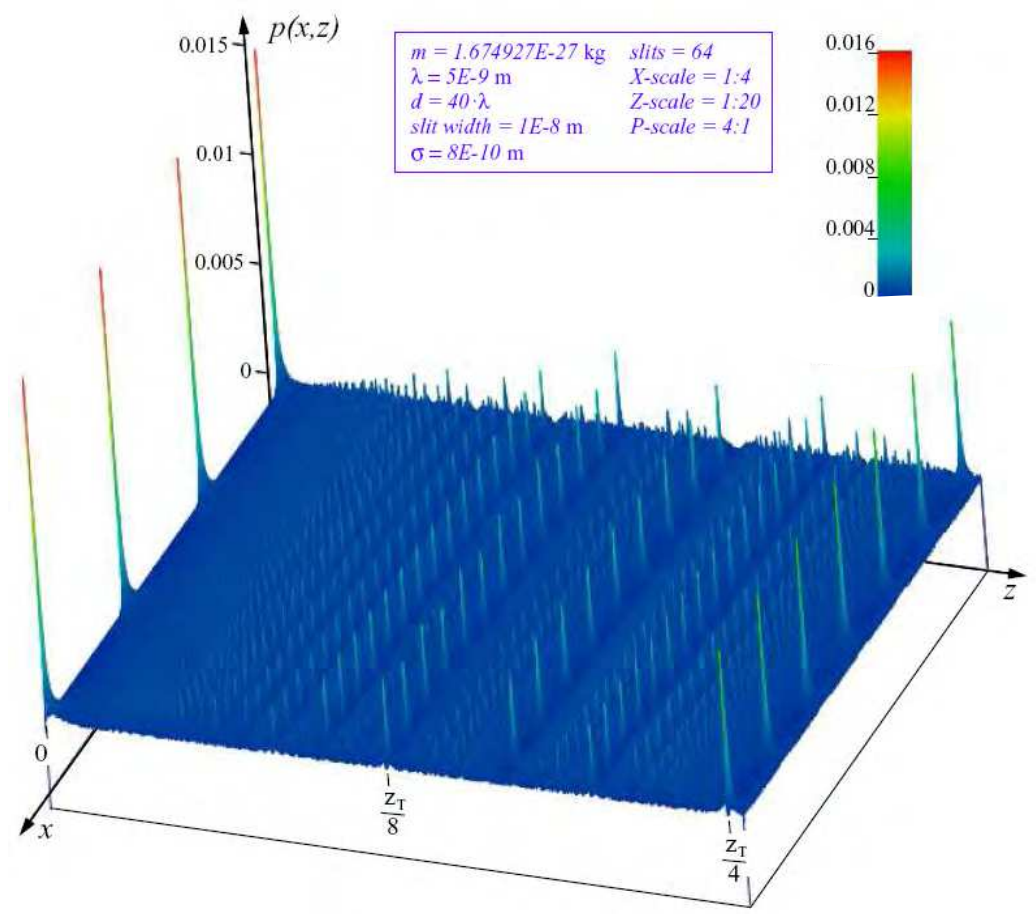

Fig. 10. Probability density distribution approaches infinite set of $\delta$-functions as $\lambda_{\mathrm{dB}} / d$ tends to zero. Parameters here are as follows $N=64, \lambda_{\mathrm{dB}}=0.5 \mathrm{~nm}$ (thermal neutrons), $d=20 \mathrm{~nm}, z_{\mathrm{T}}$ $=6400 \mathrm{~nm}$ (Sbitnev, 2009b). 
In order to reach the Berry's conditions, we take number of slits as many as possible. The ratio $\lambda_{\mathrm{dB}} / d$ should be as small as possible, as well. Given $\lambda_{\mathrm{dB}}=5 \mathrm{pm}$ and $d=500 \mathrm{~nm}$ we have the ratio $\lambda_{\mathrm{dB}} / d=10^{-5}$. It is in good agreement with the condition (b). As for number of slits, as seen in Fig. 8 the interference patterns are washed away on first some Talbot lengths. It means, that number of the slits $N=15$ is insufficient for observing of the Talbot carpet. Fig. 9 shows emergence of the Talbot carpet in the near-field in central part of the grating having 255 slits. As seen, $\mathrm{N}=255$ is sufficient number to get the Talbot carpet with perfect organization of alternation of high and low values of the density distribution.

\subsection{Bohmian trajectories}

How does particle pass through the slits? Answer to this problem is proposed in the Bohmian mechanics (Bohm, 1952a, 1952b; Bohm \& Hiley, 1982; Hiley, 2002). In the next section we will consider this solution in detail. Here we show only some particular solutions. Two divergent Bohmian trajectories drawn in blue are shown in Fig. 8. They prefer to go along dark plots (high values of the density distribution) and avoid lightcolored plots (low values of the density distribution). Fig. 9 also shows in blue a family of the Bohmian trajectories. In contrast to the trajectories shown in previous figure, here they demonstrate complex zigzag movements. The Bohmian trajectories result from solution of the guidance equation (Wyatt \& Bittner, 2003; Nikolić, 2007; Sanz \& Miret-Artês, 2007, 2008; Struyve \& Valentini, 2009)

$$
v_{x}=\dot{x}=\frac{\nabla S}{m}=\frac{\hbar}{m} \operatorname{Im}\left(|\Psi\rangle^{-1} \nabla|\Psi\rangle\right) .
$$

According to the equation (31), position $(x, z)$ of the particle in 2D space is given as follows

$$
x(t)=x_{0}+\int_{0}^{t} v_{x} d \tau, \quad z(t)=z_{0}+v_{z} t .
$$

Since we believe that longitudinal momentum, $p_{z}$, is constant in contrast to the transversal momentum $p_{x}$, the component $z$ here is calculated by simple multiplication of $v_{z}$ by $t$. In turn, velocity $v_{x}$, as seen from Eq. (31), is (a) proportional to gradient of the wave function; and (b) inversely proportional to the same wave function. It means: (a) a trajectory undergoes greatest variations in plots, where the wave function has slopes; and (b) the trajectory avoid areas, where the wave function tends to zero.

One could think that the Bohmian trajectories are physical artifacts, since they enter into a rough contradiction with the Heisenberg uncertainty principle, because of prediction in each time moment of exact values of coordinates and velocities of the particle (Bohm, 1952a, 1952b; Bohm \& Hiley1982). However, there is no here contradiction so far as the uncertainty principle refers to the measurement problem. Whereas the Bohmian trajectories are simply geodesic trajectories. At drawing the density distribution function we could use an orthogonal grid represented by geodesic trajectories and surfaces of equal phases, see, for example, Fig. 1. In the absence of intervention in a particle's history by measuring its parameters, real particle prefer to move along a geodesic trajectory. However, as soon as we undertake measurement of the particle's parameters we destroy its history. For example, if we measure position of the particle, we destroy its future history. If we measure its momentum, then we lose its past history. 
The Bohmian trajectories in Fig. 9 are seen to fulfill intricate zigzag dances. One can see, the trajectories pass through areas where the density distribution has high values and avoid areas with low its values. The particles one can guess should perform zigzag motions. However, as was noted above, the ratio $z_{\mathrm{T}}$ to $d$ is about $2.10^{5}$ and the observed pattern is within a very narrow strip. Consequently, these zigzags have very small curvatures. Vacuum fluctuations can provoke emergence of such deviations.

\section{Variational computations}

What could cause the particle to perform such a wavy and zigzag behaviors, as shown in the figures above? Possible answer could be as follows: a family of ordered slits in the screen poses itself as a quantum object that polarizes vacuum in the near-field region. The polarization, in turn, induces formation of a virtual particle's escort around of a flying real particle through the space. The escort corrects movement of the particle depending on the environment by interference of virtual particles with each other (Feynman \& Hibbs, 1965).

\subsection{Wave-particle duality, the Schrödinger equation}

In contrast to classical mechanics where a single trajectory connecting the initial and final points submits to the principle of least action, in the quantum mechanics we need to consider all possible trajectories connecting these points in order to obtain clear answer. They pass through all intermediate points belonging to a transitional set $\mathbb{R}^{3}$. All these paths should be evaluated jointly. Such a description goes back to the integral ChapmanKolmogorov equation (Ventzel, 1975):

$$
p(x, z ; t+\tau)=\int_{R^{n}} p(x, y ; t) p(y, z, \tau) d y
$$

which gives transitional probability densities of a Markovian sequence.

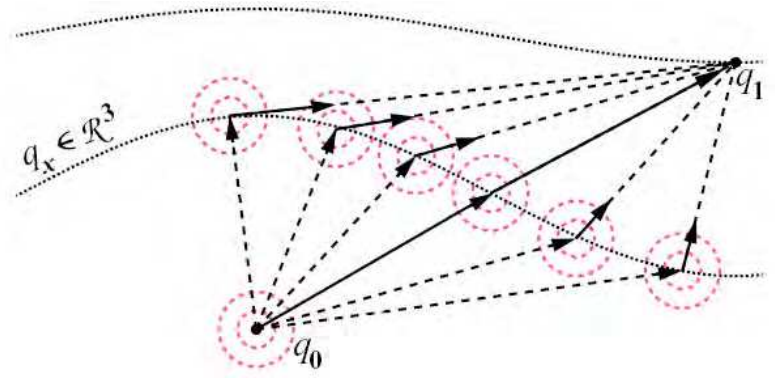

Fig. 11. Computation of all possible paths that pass from point $q_{0}$ to point $q_{1}$ through possible intermediate points $q_{x} \in \mathbb{R}^{3}$ represents a core of the path integral method. Pink circles conditionally represent radiation of Huygens waves.

Essential difference from the classical probability theory is that instead of the probabilities quantum mechanics deals with probability amplitudes containing imaginary terms. They bear information about phase shifts accumulated along paths. In that way, a transition from an initial state $\vec{q}_{0}$ to a final state $\vec{q}_{1}$ through all intermediate positions $\vec{q}_{x}$ given on a conditional set $\mathbb{R}^{3}$ (see Fig. 11) is represented by the following path integral 


$$
\psi\left(\vec{q}_{1}, \vec{q}_{0}, t+\delta t\right)=\int_{\mathcal{R}^{3}} K\left(\vec{q}_{1}, \vec{q}_{x} ; t+\delta t, t\right) \psi\left(\vec{q}_{x}, \vec{q}_{0}, t\right) \mathcal{D}^{3} q_{x}
$$

in the limit $\delta t \rightarrow 0$ and $\vec{q}_{1} \rightarrow \vec{q}_{x}$. Here symbol $\mathscr{D}^{3} q_{x}$ represents a differential element of volume in the set $R^{3}$.

Circular waves pictured by dotted circumferences in Fig. 11 illustrate working of the Huygens-Fresnel principle (Landsberg, 1957; Longhurst, 1970). The principle proclaims that each point $\vec{q}_{x}$ at an advanced wave front is, in fact, the center of a fresh disturbance and it is the source of a new wave radiation. The advancing wave as a whole may be regarded as the sum of all the secondary waves arising from points in the medium already traversed by the wave. All the secondary waves are coherent, since they are activated from the one source given in $\vec{q}_{0}$.

It is important to note, that all rays from such secondary sources represent virtual trajectories emanating from the source at $\vec{q}_{0}$ up to the point $\vec{q}_{1}$. Along with the other virtual trajectories generated by the other secondary sources, all together they create in the point $\vec{q}_{1}$ an averaged effect of contribution of these secondary sources. This averaged effect shows whether a real particle passes by this route and what probability of this event can be.

We suppose that the integral kernel

$$
K\left(\vec{q}_{1}, \vec{q}_{x} ; t+\delta t, t\right)=\frac{1}{A} \exp \left\{\frac{\mathrm{i}}{\hbar} L\left(\vec{q}_{x}, \dot{\vec{q}}_{x}\right) \delta t\right\}
$$

has a standard form of the Lagrangian (Feynman \& Hibbs, 1965)

$$
L\left(\vec{q}_{x}, \dot{\vec{q}}_{x}\right)=\frac{m}{2}\left(\frac{\vec{q}_{1}-\vec{q}_{x}}{\delta t}\right)^{2}-U\left(\vec{q}_{x}\right) .
$$

Here $U\left(\vec{q}_{x}\right)$ is a potential energy of the particle localized at the point $\vec{q}_{x} \in \mathbb{R}^{3}$. And $\left(\vec{q}_{1}-\vec{q}_{x}\right) / \delta t$ is a velocity $\dot{\vec{q}}_{x}$ attached to the same point $\vec{q}_{x}$ and oriented in the direction of the point $\vec{q}_{1}$.

The next step is to expand terms, ingoing into the integral (34), into Taylor series. The wave function written at the left is expanded up to the first term

$$
\psi\left(\vec{q}_{1}, \vec{q}_{0}, t+\delta t\right) \approx \psi\left(\vec{q}_{1}, \vec{q}_{0}, t\right)+\frac{\partial \psi}{\partial t} \delta t .
$$

As for the terms under the integral, here we preliminarily make some transformations. We define a small increment

$$
\vec{\xi}=\vec{q}_{1}-\vec{q}_{x} \Rightarrow \mathcal{D}^{3} q_{x}=-\mathcal{D}^{3} \xi
$$

The Lagrangian (36) is written as

$$
L\left(\vec{q}_{x}, \dot{\vec{q}}_{x}\right)=\frac{m}{2} \frac{\xi^{2}}{\delta t^{2}}-U\left(\vec{q}_{1}-\vec{\xi}\right) .
$$

Here the potential energy $U\left(\vec{q}_{1}-\vec{\xi}\right)$ is subjected to expansion into the Taylor series by the small parameter $\vec{\xi}$. The under integral wave function $\psi\left(\vec{q}_{x}, \vec{q}_{0}, t\right)=\psi\left(\vec{q}_{1}-\vec{\xi}, \vec{q}_{0}, t\right)$ is subjected to expansion into the Taylor series up to the second terms of the expansion 


$$
\psi\left(\vec{q}_{1}-\vec{\xi}, \vec{q}_{0}, t\right) \approx \psi\left(\vec{q}_{1}, \vec{q}_{0}, t\right)-(\nabla \psi \cdot \vec{\xi})+\nabla^{2} \psi \cdot \xi^{2} / 2 .
$$

Taking into account the expressions (37)-(40) and substituting theirs into Eq. (34) we get

$$
\begin{gathered}
\psi\left(\vec{q}_{1}, \vec{q}_{0}, t\right)+\frac{\partial \psi}{\partial t} \delta t=-\frac{1}{A} \int_{R^{3}} \exp \left\{\frac{\mathrm{i}}{\hbar} \frac{m}{2} \frac{\xi^{2}}{\delta t}\right\}\left(1-\frac{\mathrm{i}}{\hbar}\left(U\left(\vec{q}_{1}\right)-(\nabla U \cdot \vec{\xi})+\Delta U \cdot \xi^{2} / 2\right) \delta t\right) \\
\left(\psi\left(\vec{q}_{1}, \vec{q}_{0}, t\right)-(\nabla \psi \cdot \vec{\xi})+\nabla^{2} \psi \cdot \xi^{2} / 2\right) \mathcal{D}^{3} \xi
\end{gathered}
$$

One can see that the term $\psi\left(\vec{q}_{1}, \vec{q}_{0}, t\right)$ is presented from both the left side and from the right side. These both term can remove each other, if the right part will satisfy the following condition

$$
-\frac{1}{A} \int_{\mathbb{R}^{3}} \exp \left\{\frac{\mathrm{i}}{\hbar} \frac{m}{2} \frac{\xi^{2}}{\delta t}\right\} \mathcal{D}^{3} \xi=-\frac{1}{A}\left(\frac{2 \pi \mathrm{i} \hbar \delta t}{m}\right)^{3 / 2}=1
$$

From here it follows

$$
A=-\left(\frac{2 \pi \mathrm{i} \hbar \delta t}{m}\right)^{3 / 2}
$$

The power 3 arises here because that the integration is fulfilled on the 3-dimensional set $\mathbb{R}^{3}$. It would be desirable also to integrate the terms $(\nabla \psi \cdot \vec{\xi})$ and $\nabla^{2} \psi \cdot \xi^{2} / 2$ existing in the integral (41). With this aim in the mind, we mention the following two integrals (Feynman \& Hibbs, 1965)

$$
\frac{1}{A} \int_{\mathbb{R}^{3}} \exp \left\{\frac{\mathrm{i}}{\hbar} \frac{m}{2} \frac{\xi^{2}}{\delta t}\right\} \vec{\xi} \mathscr{D}^{3} \xi=0
$$

and

$$
\frac{1}{A} \int_{\mathbb{R}^{3}} \exp \left\{\frac{\mathrm{i}}{\hbar} \frac{m}{2} \frac{\xi^{2}}{\delta t}\right\} \xi^{2} \mathscr{D}^{3} \xi=\frac{\mathrm{i} \hbar}{m} \delta t .
$$

In accordance with the first integral, contributions of the terms $\nabla \psi$ and $\nabla U$ in the expression (41) disappear. Whereas, the terms with multiplier $\xi^{2} / 2$ gains the factor $(\mathrm{i} \hbar \delta t / m) / 2$.

Taking into account the above stated expressions, let us rewrite Eq.( 41)

$$
\frac{\partial \psi}{\partial t} \delta t=\mathrm{i} \frac{\hbar \delta t}{2 m} \Delta \psi-\mathrm{i} \frac{\delta t}{\hbar} U\left(\vec{q}_{1}\right) \psi\left(\vec{q}_{1}, \vec{q}_{0}, t\right)+\frac{\delta t^{2}}{2 m} \Delta U\left(\vec{q}_{1}\right) \psi\left(\vec{q}_{1}, \vec{q}_{0}, t\right) .
$$

The last term contains the factor $\delta t^{2}$ due to which contribution of this term to this equation is abolished in contrast with other terms as $\delta t \rightarrow 0$. By omitting this term, we come to the Schrödinger equation 


$$
\mathrm{i} \hbar \frac{\partial \psi}{\partial t}=-\frac{\hbar^{2}}{2 m} \Delta \psi+U\left(\vec{q}_{1}\right) \psi
$$

describing the function $\psi$, wave function, in the configuration space $\mathbb{R}^{3}$. The subscript 1 here can be dropped.

\subsection{The Bohmian decomposition}

Let us examine the Schrödinger equation (47) by substituting the wave function $\psi$ in the following form:

$$
\psi(\vec{q}, \vec{p}, t)=\sqrt{\rho(\vec{q}, \vec{p}, t)} \exp \{\mathrm{i} S(\vec{q}, \vec{p}, t) / \hbar\}=R(\vec{q}, \vec{p}, t) \exp \{\mathrm{i} S(\vec{q}, \vec{p}, t) / \hbar\} .
$$

Here functions $S(\vec{q}, \vec{p}, t)$ and $\rho(\vec{q}, \vec{p}, t)$ are real functions of their variables $\vec{q}, \vec{p}$, and $t$. The first function is the action which was mentioned earlier. And the second function is the probability density distribution defined as follows

$$
\rho(\vec{q}, \vec{p}, t)=|\psi|^{2}=\psi^{*} \psi
$$

Here we will consider the decomposition in a general view, i.e., the variables $\vec{q}=\left(q_{1}, q_{2}, \cdots, q_{N}\right)$ and $\vec{p}=\left(p_{1}, p_{2}, \cdots, p_{N}\right)$ are those representing the quantum system in $2 N-$ dimensional phase space. It means, in particular, that there are several particles which can be considered in this space as one generalized particle.

By substituting the wave function $\psi(\vec{q}, \vec{p}, t)$ into the Schrödinger equation (47) we obtain

$$
\begin{gathered}
\underbrace{-\frac{\partial S}{\partial t} \cdot \psi}_{(\mathrm{a})} \underbrace{+\mathrm{i} \hbar \frac{1}{2 \rho} \frac{\partial \rho}{\partial t} \cdot \psi}_{(\mathrm{b})}=\underbrace{\frac{1}{2 m}(\nabla S)^{2} \cdot \psi+U(\vec{q}) \cdot \psi}_{(\mathrm{a})} \\
\underbrace{-\frac{\mathrm{i} \hbar}{2 m} \nabla^{2} S \cdot \psi-\frac{\mathrm{i} \hbar}{2 m}\left(\frac{1}{\rho} \nabla \rho\right)(\nabla S) \cdot \psi}_{\text {(b) }} \\
=\underbrace{-\frac{\hbar^{2}}{2 m}\left(\frac{1}{2 \rho} \nabla^{2} \rho\right) \cdot \psi+\frac{\hbar^{2}}{2 m}\left(\frac{1}{2 \rho} \nabla \rho\right)^{2} \cdot \psi}_{(\mathrm{c})}
\end{gathered}
$$

Operators of gradient, $\nabla$, and laplacian, $\nabla^{2}$, read

$$
\nabla=\left\{\frac{\partial}{\partial q_{1}} i_{1}, \frac{\partial}{\partial q_{2}} i_{2}, \cdots, \frac{\partial}{\partial q_{N}} i_{N}\right\}, \quad \nabla^{2}=\left\{\frac{\partial^{2}}{\partial q_{1}^{2}}+\frac{\partial^{2}}{\partial q_{2}^{2}}+\cdots+\frac{\partial^{2}}{\partial q_{N}^{2}}\right\} .
$$

A set $\left\{i_{1}, i_{2}, \ldots, i_{N}\right\}$ represents orthonormal basis of $N$-dimensional state space $S^{N}$. The orthonormality means that $i_{k} \cdot i_{j}=\delta_{k, j}$ for all $k, j$ ranging 1 to $N$.

Collecting together real terms (a) and (c), and separately imaginary terms (b) in Eq. (50) we obtain two coupled equations for real functions $S(\vec{q}, \vec{p}, t)$ and $\rho(\vec{q}, \vec{p}, t)$

$$
-\frac{\partial S}{\partial t}=\frac{1}{2 m}(\nabla S)^{2}+U(\vec{q}) \underbrace{-\frac{\hbar^{2}}{2 m}\left[\frac{\nabla^{2} \rho}{2 \rho}-\left(\frac{\nabla \rho}{2 \rho}\right)^{2}\right]}_{(\mathrm{c})},
$$




$$
-\frac{\partial \rho}{\partial t}=\nabla\left(\rho \frac{\nabla S}{m}\right)
$$

A term

$$
Q=-\frac{\hbar^{2}}{2 m}\left[\frac{\nabla^{2} \rho}{2 \rho}-\left(\frac{\nabla \rho}{2 v}\right)^{2}\right]=-\frac{\hbar^{2}}{2 m} \frac{\nabla^{2} R}{R}
$$

enveloped by brace (c) in Eq. (52) is the quantum potential. It evaluates a measure of curvature of the $\mathrm{N}$-dimensional state space induced by a prepared physical scene consisting of sources, detectors, and other experimental devices. Equations, (52) and (53), are seen to be the coupled pair of nonlinear partial differential equations. The first of the two equations, Eq. (52), is the Hamilton-Jacobi equation modified by the quantum potential $Q(\vec{q}, t)$. The second equation, Eq. (53), is the continuity equation. In the above equations we define the following computations

$$
\vec{p}=m \vec{v}=\nabla S
$$

and

$$
\frac{1}{2 m}(\nabla S)^{2}=\frac{1}{2 m} p^{2}
$$

Here $\vec{p}$ is momentum of the particle, $\vec{v}$ is its velocity, and the last equation represents kinetic energy of the particle.

Equation (52) states that total particle energy is the sum of the kinetic energy, potential energy, and the quantum potential (Hiley, 2002). Equation (53), in turn, is interpreted as the continuity equation for probability density $\rho(\vec{q}, \vec{p}, t)$. It says that all individual trajectories demonstrate collective behavior like a liquid flux (Madelung, 1926; Wyatt, 2005), perhaps, superconductive one. We shall see further, that the quantum potential $Q(\vec{q}, \vec{p}, t)$ introduces corrections both in the kinetic energy and in the potential energy of the particle.

\subsubsection{The quantum potential as an information channel}

According to the observation

$$
\rho^{-1} \nabla \rho=\nabla \ln (\rho)
$$

we can rewrite the quantum potential by the following way

$$
Q(\vec{q}, t)=\frac{\hbar^{2}}{2 m}\left[\left(\frac{1}{2 \rho} \nabla \rho\right)^{2}-\frac{1}{2}\left(\frac{1}{\rho} \nabla\left(\rho \cdot \frac{1}{\rho} \nabla \rho\right)\right)\right]=-\frac{\hbar^{2}}{2 m}\left(\frac{1}{2} \nabla \ln (\rho)\right)^{2}-\frac{\hbar^{2}}{2 m}\left(\frac{1}{2} \nabla^{2} \ln (\rho)\right) .
$$

Define a logarithmic function

$$
S_{Q}(\vec{q}, \vec{p}, t)=-\frac{1}{2} \ln (\rho(\vec{q}, \vec{p}, t))
$$


to be called further quantum entropy. It is like to the Boltzmann entropy. However if the Boltzmann entropy characterizes degree of order and chaos of classical gases, the quantum entropy evaluates analogous quality of the quantum liquid mentioned above. To be more defined, one can imagine the quantum liquid as ensemble of partially ordered virtual vortices (particle-antiparticle pairs) within vacuum. For example, such virtual vortices may be presented by spinning electron-positron pairs.

Substituting (59) into Eq. (58) we find that the quantum potential can be expressed in terms of this function

$$
Q(\vec{q}, \vec{p}, t)=\underbrace{-\frac{\hbar^{2}}{2 m}\left(\nabla S_{Q}\right)^{2}}_{(\text {a) }}+\underbrace{\frac{\hbar^{2}}{2 m} \nabla^{2} S_{Q}}_{(\text {b) }} .
$$

It should be noted, that the term $-S_{Q}$ (negative $S_{Q}$ ) is named $C$-amplitude in (Bittner, 2003; Wyatt, 2005; Wyatt \& Bittner, 2003). Here the term enveloped by brace (a) is viewed as the quantum corrector of the kinetic energy. And the term enveloped by brace (b) corrects the potential energy. Namely, substituting into Eq. (52) we obtain

$$
-\frac{\partial S}{\partial t}=\underbrace{\frac{1}{2 m}(\nabla S)^{2}-\frac{\hbar^{2}}{2 m}\left(\nabla S_{Q}\right)^{2}}_{(\text {a) }}+\underbrace{U(\vec{q})+\frac{\hbar^{2}}{2 m} \nabla^{2} S_{Q}}_{(\mathrm{b})} .
$$

In this equation the terms enveloped by brace (a) relate to the kinetic energy of the particle, and those enveloped by brace (b) relate to its potential energy.

Substituting also $S_{Q}$ in the continuity equation (53) instead of $\rho$ we obtain the entropy balance equation

$$
\frac{\partial S_{Q}}{\partial t}=-\left(\vec{v} \cdot \nabla S_{Q}\right)+\frac{1}{2}(\nabla \vec{v})
$$

Here $\vec{v}=\nabla S / m$ is a particle speed. The rightmost term, $(\nabla \vec{v})$, describes a rate of the entropy flow produced by spatial divergence of the speed due to curvature of the $N$-dimensional state space. This term is nonzero in regions where the particle changes direction of movement.

\section{Beyond the Bohm's insight into QM}

Pair of the equations, the modified HJ equation (61) and the entropy balance equation (62), describes behavior of the quantum particle, subject to influence of the quantum entropy. Let us now multiply Eq. (62) by the factor $-\mathrm{i} \hbar$ and add the result to Eq. (61). We obtain

$$
-\frac{\partial \mathbf{S}}{\partial t}=\underbrace{\frac{1}{2 m}(\nabla S)^{2}+\mathrm{i} \hbar \frac{1}{m}\left(\nabla S \cdot \nabla S_{Q}\right)-\frac{\hbar^{2}}{2 m}\left(\nabla S_{Q}\right)^{2}}_{\text {(a) }}+\underbrace{U(\vec{q})-\mathrm{i} \hbar \frac{1}{2}(\nabla \vec{v})+\frac{\hbar^{2}}{2 m} \nabla^{2} S_{Q}}_{\text {(b) }} .
$$

Here $S$ is sum of the action $S$ and the quantum entropy $S_{Q}$ (complexified action)

$$
\mathbf{S}=S+\mathrm{i} \hbar S_{Q}
$$


Terms enveloped by brace (a) can be rewritten as gradient of the squared complexified action

$$
\frac{1}{2 m}(\nabla \mathbf{S})^{2}=\frac{1}{2 m}(\nabla S)^{2}+\mathrm{i} \hbar \frac{1}{m}\left(\nabla S \cdot \nabla S_{Q}\right)-\frac{\hbar^{2}}{2 m}\left(\nabla S_{Q}\right)^{2} .
$$

As for the terms enveloped by brace (b) they could stem from expansion into the Taylor's series of the potential energy extended previously to a complex space, like a complex extension, for example, in (Poirier, 2008). In our case, the potential function is extended in the complex space, which has a small broadening into imaginary sector. Let us expand into the Taylor's series the potential function that has a complex argument

$$
U(\vec{q}+\mathrm{i} \varepsilon) \approx U(\vec{q})+\mathrm{i}(\vec{\varepsilon} \cdot \nabla U(\vec{q}))-\frac{\varepsilon^{2}}{2} \nabla^{2} U(\vec{q})+\ldots
$$

Now we will examine the last two terms. Here a small vector $\vec{\varepsilon}$ has dimensionality of length. But it should contain also the Planck constant, $\hbar$, in order to reproduce the second and third terms enveloped by brace (b) in Eq. (63). A minimal representation of this vector can be as follows

$$
\vec{\varepsilon}=\frac{\hbar}{2 m} S_{\mathrm{B}} \vec{n}
$$

Here $\vec{n}$ is unit vector pointing direction of the small increment, $m$ is the particle mass, and $s_{B}$ is universal constant, "reverse velocity",

$$
s_{\mathrm{B}}=4 \pi \varepsilon_{0} \frac{\hbar}{e^{2}} \approx 4.57 \times 10^{-7}[\mathrm{~s} / \mathrm{m}]
$$

Here $e \approx-1.6 \times 10^{-19}[\mathrm{C}]$ is the elementary charge carried by a single electron and $\varepsilon_{0} \approx 8.854 \times 10^{-12}\left[\mathrm{C}^{2} \mathrm{~N}^{-1} \mathrm{~m}^{-2}\right]$ is the vacuum permittivity. The reverse velocity measures time required for traversing unit of a distance. Such a distance can be perimeter of orbit (Poluyan, 2005) at oscillating electron around. Observe that $r_{\mathrm{B}}=s_{\mathrm{B}} \hbar / m=4 \pi \varepsilon_{0} \hbar^{2} / m e^{2}$ is value of the electron radius under its travelling on first orbit around the nucleus (Dirac, 1982). In our case it can be an effective radius of electron-positron pair under their virtual revolution about the mass center on the first orbit. From the above it follows, that $v_{\mathrm{B}}=1 / \mathrm{s}_{\mathrm{B}} \approx 2.188 \times 10^{6} \mathrm{~m} / \mathrm{s}$ is the Bohr velocity of electron oscillating on the first orbit about the mass center, and $r_{\mathrm{B}}=\hbar / m v_{\mathrm{B}} \approx 0.529 \times 10^{-10} \mathrm{~m}$ is the Bohr radius of this orbit. Here $m v_{\mathrm{B}}$ is the electron momentum.

In light of these remarks, we can rewrite the expansion (66) in the following form

$$
\begin{aligned}
U(\vec{q}+\mathrm{i} \varepsilon) & \approx U(\vec{q})+\underbrace{\mathrm{i} \hbar\left(\vec{n} \cdot\left(\frac{s_{\mathrm{B}}}{2 m} \nabla U(\vec{q})\right)\right)}_{\left(\mathrm{b}_{1}\right)} \underbrace{-\frac{\hbar^{2}}{2 m}\left(\frac{s_{\mathrm{B}}^{2}}{2 m} \nabla^{2} U(\vec{q})\right)}_{\left(\mathrm{b}_{2}\right)} . \\
& =U(\vec{q})+\underbrace{\frac{\mathrm{i}\left(\vec{n} r_{\mathrm{B}} \cdot \nabla U(\vec{q})\right)}{2} \underbrace{\frac{1}{4} r_{\mathrm{B}}^{2} \nabla^{2} U(\vec{q})}_{\left(\mathrm{b}_{2}\right)} .}_{\left(\mathrm{b}_{1}\right)} .
\end{aligned}
$$


A term enveloped by brace $\left(b_{1}\right)$ contains unit vector $\vec{n}$ that points out direction of the imaginary broadening. A force $\vec{F}=-\nabla U(\vec{q})$ multiplied by $\vec{n} r_{\mathrm{B}}$ is elementary work performed at displacement on a length $r_{B}$ along direction $\vec{n}$. The elementary work divided into $\hbar$ is a rate of variation of the particle velocity per unit length, i.e., it represents divergence of the velocity, $\nabla \vec{v}$. So, the term enveloped by brace $\left(b_{1}\right)$ can be rewritten in the following form

$$
\left(\mathrm{b}_{1}\right): \frac{1}{\hbar}\left(\vec{n} r_{\mathrm{B}} \cdot \nabla U(\vec{q})\right)=-(\nabla \cdot \vec{v}) .
$$

As for the term $\left(s_{B}^{2} / 2 m\right) \cdot U(\vec{q})=\left(1 / 2 m v_{B}^{2}\right) \cdot U(\vec{q})$ which is placed over brace $\left(\mathrm{b}_{2}\right)$ in Eq. (69) it is dimensionless. Accurate to an additive dimensionless function $a \vec{q}^{2}+(\vec{b} \vec{q})+c$ this term is comparable with $S_{\mathrm{Q}}$, i.e., with $\ln (\rho)$. Taking into account that $s_{\mathrm{B}}=1 / v_{\mathrm{B}}$ we proclaim

$$
\left(\mathrm{b}_{2}\right):-\left(\frac{1}{2 m v_{\mathrm{B}}^{2}} \nabla^{2} U(\vec{q})\right)=\frac{1}{2 m v_{\mathrm{B}}^{2}}(\nabla \vec{F})=\nabla^{2} S_{Q} .
$$

Thus, a value of the Laplacian of $U(\vec{q})$ at the point $\vec{q}$ can be interpreted as the density of sources (sinks) of the potential vector field $\vec{F}=-\nabla U(\vec{q})$ at this point. Accurate to the denominator $2 m v_{\mathrm{B}}^{2}$, it is proportional to the Laplacian of the quantum entropy $S_{Q}$.

We have defined the corrections (70) and (71) by extending coordinates of the real 3D space into imaginary domain on the value $\varepsilon$. It is equal to about the Bohr radius of the first orbit of the electron-positron virtual pair, $r_{\mathrm{B}} \approx 5.292 \times 10^{-11} \mathrm{~m}$. Energy of this pair is much smaller of the energy creating two real particles from the vacuum. Therefore such a shift, $\varepsilon=r_{\mathrm{B}} / 2$, can be considered as a virtual small shift to the imaginary domain.

Now we can define complexified momentum

$$
\vec{P}=m \dot{\vec{Q}}=\nabla \mathbf{S}=\nabla S+\mathrm{i} \hbar \nabla S_{Q}
$$

and complexified coordinate

$$
\vec{Q}=\vec{q}+\mathrm{i} \vec{\varepsilon}
$$

as extended representations of the real vectors $\vec{p}$ and $\vec{q}$. The complexified momentum $\vec{P}$ differs from momentum $\vec{p}$ by additional imaginary term $\hbar \nabla S_{Q}$. And the complexified coordinate $\vec{Q}$ differs from real coordinate $\vec{q}$ by the small imaginary vector (67). Now we can rewrite Eq. (63) as complexified the Hamilton-Jacobi equation:

$$
-\frac{\partial \mathbf{S}}{\partial t}=\frac{1}{2 m}(\nabla \mathbf{S})^{2}+U(\vec{q}+\mathrm{i} \varepsilon)=\mathcal{H}(\vec{Q}, \vec{P} ; t)
$$

Here $H(\vec{Q}, \vec{P} ; t)$ is a complexified Hamiltonian.

The total derivative of the complex action reads

$$
\frac{d \mathbf{S}}{d t}=\frac{\partial \mathbf{S}}{\partial t}+\sum_{n=1}^{N} \frac{\partial S}{\partial Q_{n}} \frac{d Q_{n}}{d t}=\frac{\partial \mathbf{S}}{\partial t}+\sum_{n=1}^{N} \mathscr{P}_{n} \dot{Q}_{n}
$$


where complex derivative is (see Ch.2 in (Titchmarsh, 1976))

$$
\frac{\partial \mathbf{S}}{\partial Q_{n}}=\frac{\partial S}{\partial q_{n}}+\mathrm{i} \hbar \frac{\partial S_{Q}}{\partial q_{n}}=\mathscr{P}_{n} .
$$

Combining Eq. (75) with (76) we come to the Legendre's dual transformation (Lanczos, 1970) that binds the Hamiltonian $\mathcal{H}$ and the Lagrangian $\mathcal{L}$, and conversely:

$$
\frac{d \mathbf{S}}{d t}=-\mathcal{H}(\overrightarrow{\mathrm{Q}}, \overrightarrow{\mathcal{P}} ; t)+\sum_{n=1}^{N} \mathscr{P}_{n} \dot{\mathrm{Q}}_{n}=\mathcal{L}(\overrightarrow{\mathrm{Q}}, \dot{\vec{Q}} ; t) .
$$

We summarize this section by collecting formulas of the complexified Hamiltonian and

\begin{tabular}{|c|c|}
\hline Variables: & Variables : \\
\hline Coordinate: $\quad \vec{Q}=\vec{q}+\mathrm{i} \frac{\hbar}{2 m v_{\mathrm{B}}} \vec{n}$ & Coordinate: $\quad \vec{Q}=\vec{q}+\mathrm{i} \vec{\varepsilon}=\vec{q}+\mathrm{i} \frac{\hbar}{2 m v_{\mathrm{B}}} \vec{n}$ \\
\hline$\vec{P}=\vec{p}+\mathbf{i} \hbar \nabla S_{Q}$ & Velocity: $\quad \dot{\vec{Q}}=\dot{\vec{q}}+\mathrm{i} \dot{\vec{\varepsilon}}=\dot{\vec{q}}+\mathrm{i} \frac{\hbar}{2 m v_{\mathrm{B}}} \dot{\vec{n}}$ \\
\hline Hamiltonian function: & Lagrangian function: \\
\hline$H(\vec{Q}, \vec{P}, t)=\sum_{n=1}^{N} \mathscr{P}_{n} \dot{Q}_{n}-L(\vec{Q}, \dot{\vec{Q}}, t)$ & $L(\vec{Q}, \dot{\vec{Q}}, t)=\sum_{n=1}^{N} \mathscr{P}_{n} \dot{Q}_{n}-H(\vec{Q}, \vec{P}, t)$ \\
\hline$\frac{\partial H}{\partial P_{n}}=\dot{Q}_{n}$ & $\frac{\partial L}{\partial \dot{Q}_{n}}=P_{n}$ \\
\hline$\frac{\partial H}{\partial Q_{n}}=-\dot{P}_{n}$ & $\frac{\partial L}{\partial Q_{n}}=\dot{P}_{n}$ \\
\hline
\end{tabular}
Lagrangian mechanics via the Legendre's dual transformations in Table 2:

Table 2. The Legendre's dual transformations.

The Lagrangian equations of motions and the Legendre's transformations are invariant under the above fulfilled imaginary extension of the real momenta, $p_{n}$, and the real velocities, $v_{n}, n=1,2, \ldots N$. It should be noted, that the Hamiltonian function is quadratic in the momenta, $\mathscr{P}_{n}$, and the Lagrangian function is quadratic in the velocities, $\dot{Q}_{n}$. A conservation law in this case unifies conservation of energy represented by real part, $\operatorname{Re}[\mathcal{H}(\overrightarrow{\mathrm{Q}}, \overrightarrow{\mathcal{P}} ; t)]$, and the entropy balance (62) represented by imaginary part, $\operatorname{Im}[\mathcal{H}(\overrightarrow{\mathrm{Q}}, \overrightarrow{\mathcal{P}} ; t)]$. One can see from definition of the complexified velocity presented in this table, that tip of the small vector $\dot{\vec{\varepsilon}}$ performs rotating movements on the sphere of the Bohr radius $r_{\mathrm{B}}=$ $\hbar / 2 m v_{\mathrm{B}}$. This radius is about $5.3 \times 10^{-11} \mathrm{~m}$ for the electron-positron pair dancing on the first, virtual, orbit. Energy of this pair, $E=\hbar v_{\mathrm{B}} / r_{\mathrm{B}} e=27 \mathrm{~V}$, lies much below energy of the 
electron-positron creation, $E=\hbar c / \lambda_{C} e=\hbar\left(v_{\mathrm{B}} a^{-1}\right) /\left(r_{\mathrm{B}} a\right) e=511 \mathrm{kV}$. Here $e$ is the electric charge, $c$ is the speed of light, $\lambda_{C}$ is the Compton wavelength, and $\alpha \simeq 1 / 137$ is the fine structure constant. From here it is seen, that there is a wide scope of energies for correcting movement of real particle by virtual ones.

\section{Concluding remarks}

Classical mechanics supposes a principle possibility of simultaneous measuring both coordinates $(x, y, z)$ of material objects and their relative velocities $\left(v_{x}, v_{y}, v_{z}\right)$. In the beginning of 20th century scientists call in question such simultaneous measurements. Methods of the classical mechanics cease to give correct results on microscopic level. Instead of the classical equations describing behavior of a classical body, equations of quantum mechanics deal with wave functions that encompass behavior of any particle belonging to the same ensemble of coherent particles. The wave function bears information about distribution of particles that populate a space-time prepared by experimenter. It is said in that case, that it is a guidance function. It contains both the action $S$ and a quantum entropy $S_{Q}$ (logarithm of the density distribution with negative sign) in the following manner

$$
|\Psi(\vec{Q}, \vec{P}, t)\rangle=\exp \left\{\mathrm{i} S / \hbar-S_{Q}\right\} .
$$

In contrast to the classical mechanics here the action traces all routes weighted with the factor proportional to the density distribution $\rho=\exp \left\{-S_{Q}\right\}$.

Wave functions within the same physical scene (the scene is represented by particle sources, detectors, and different physical devices placed between them) obey to superposition principle. Namely, sum of the wave functions is again a wave function that bears information about organization of the physical scene. At measurements we detect interference effects that are conditioned by a specific physical scene. There is no collapse of a wave function at the moment of detecting particle. Information relating to the physical scene exists until destruction of the scene happens. It can be picked up by a new particle again as soon as the particle will be generated by the source. The physical scene prepared by experimenter defines a space-time volume in which particles emitted by sources evolve. The Schrödinger equation (Schrödinger, 1926) gives formulas that determine a probable evolution of the particles within the space-time predefined by boundary conditions of a task. Madelung (Madelung, 1926) and then Bohm (Bohm, 1952a, 1952a) have demonstrated that behind this new equation of quantum mechanics (Schrödinger, 1926), classical equations, Hamilton-Jacobi equations together with the continuity equation, can be discerned. In contrast with the classical equations here a new term emerges - the quantum potential. According to the Madelung's views, the wave function simulates laminar flow of a "fluid" along geodesic paths, named further the Bohmian trajectories. Equiphase surfaces, in turn, are represented by secant surfaces of the trajectory's bundles. Because of these findings we cannot nowadays consider the space-time with the same point of view how it was formulated by thinkers of 17th century. The quantum potential compels to expand the 3D coordinate space onto the imaginary sector by unification the action $S$ and the quantum entropy $S_{Q}$, that is, by introducing a complex action $S+\mathrm{i} \hbar S_{Q}$. One way to envisage such a complex space is to imagine a hose-pipe. From a long distance it looks like a one dimensional line. But a closer inspection reveals that every point on the line is in fact a circle. It determines the unitary group $\mathrm{U}(1)$, which generates the term $\exp \{$ i $S / \hbar\}$ - a main term in the Feynman path integral. 


\section{Acknowledgement}

Author thanks Miss Pipa (administrator of Quantum Portal) for preparing programs, that permitted to calculate and prepare Figs. 5, 8, and 9.

\section{References}

Andresse, C. D. (2005). Huygens The Man Behind the Principle, Cambridge University Press, Cambridge

Arndt, M., Hackermüller, L. \& Reiger, E. (2005). Interferometry with large molecules: Exploration of coherence, decoherence and novel beam methods. Brazilian Journal of Physics, Vol. 35(No. 2A), 216-223

Berry, M. (1996). Quantum fractals in boxes. J. Phys. A: Math. Gen., Vol. 29, 6617-6629

Berry, M. (1997). Quantum and optical arithmetic and fractals. In: The Mathematical beauty of Physics, J.-M. Drouffe \& J.-B. Zuber, pp. 281-294, World Scientific, Singapore

Berry, M. \& Klein, S. (1996) Integer, fractional and fractal Talbot effects, Journal of Modern Optics, Vol. 43(No. 10), 2139--2164

Berry, M., Marzoli, L. \& Schleich, W. (2001). Quantum carpets, carpets of light. Physics World, (6), 39-44

Bittner, E. R. (2003). Quantum initial value representations using approximate Bohmian trajectories. http:/ /arXiv.org/abs/quant-ph/0304012

Bohm, D. (1951). Quantum theory, Prentice Hall, N. Y.

Bohm, D. (1952a). A suggested interpretation of the quantum theory in terms of "hidden variables", I. Physical Review, Vol. 85, 166--179

Bohm, D. (1952b). A suggested interpretation of the quantum theory in terms of "hidden variables", II. Physical Review, Vol. 85, 180-193

Bohm, D. J. \& Hiley, B. J. (1982). The de Broglie pilot wave theory and the further development of new insights arising out of it. Foundations of Physies, Vol. 12(No. 10), $1001-1016$

Born, M. \& Wolf, E. (1999). Principles of Optics, Cambridge University Press, Cambridge

Brezger, B., Hackermüller, L., Uttenthaler, S., Petschinka, J., Arndt, M. \& Zeilinger, A. (2002). Matter-wave interferometer for large molecules. Phys. Rev. Lett., Vol. 88, 100404

Brezger, B., Arndt, M. \& Zeilinger, A. (2003). Concepts for near-field interferometers with large molecules. J. Opt. B: Quantum Semiclass. Opt. , Vol. 5(No. 2), S82--S89

Dirac, P. A. M. (1933). The Lagrangian in quantum mechanics, Physikalische Zeitschrift der Sowjetunion, Vol. 3, 64-72

Dirac, P. A. M. (1945). On the analogy between classical and quantum mechanics, Phys. Rev. Lett., Vol. 17(Nos. 2 \& 3), 195-199

Dirac, P. A. M. (1982). Principles of Quantum Mechanics. Oxford University Press, Oxford, 4nd edition

Feynman, R. P. (1948). Space-time approach to non-relativistic quantum mechanics, Rev. Mod. Phys.., Vol. 20, 367-387

Feynman, R. P. \& Hibbs, A. (1965). Quantum mechanics and path integrals, McGraw Hill, N. Y. Gerlich, S., Eibenberger, S., Tomandl, M., Nimmrichter, S., Hornberger, K., Fagan, P. J., Tuxen, J., Mayor, M. \& Arndt, M. (2011). Quantum interference of large organic molecules. Nature Communications, Vol. 2 (No. 263) 
Grosche, C. (1993). An introduction into the Feynman path integral. http://arXiv.org/abs/hepth/9302097

Hackermüller, L., Hornberger, K., Brezger, B., Zeilinger, A. \& Arndt, M. (2003). Decoherence in a Talbot Lau interferometer: the influence of molecular scattering. Appl. Phys. B, Vol. 77, 781-787

Hackermüller, L., Hornberger, K., Brezger, B., Zeilinger, A. \& Arndt, M. (2004). Decoherence of matter waves by thermal emission of radiation. Nature, Vol. 427, 711-714

Hiley, B. J. (2002). From the Heisenberg picture to Bohm: a new perspective on active information and its relation to Shannon information. In: Quantum Theory: reconsideration of foundations, Proc. Int. Conf., A. Khrennikov, pp. 1--24, Växjö University Press, June 2001, Sweden

Juffmann, T., Truppe, S., Geyer, P., Major, A. G., Deachapunya, S., Ulbricht, H. \& Arndt, M. (2009). Wave and particle in molecular interference lithography. Phys. Rev. Lett., Vol. 103, 263601.

Kac, M. (1957). On some connections between probability theory and differential and integral equations, Proc. Second Berkeley Symposium on Mathematical Statistics and Probability, pp .189-215, Berkeley, USA

Lanczos, C. (1970). The variational principles of mechanics, Dover Publ., Inc., N. Y.

Landsberg, G. S. (1957). Optika, Moscow-Leningrad, 4nd edition

Longhurst, R. S. (1970). Geometrical and Physical Optics. Longmans, London, 2nd edition

MacKenzie, R. (2000). Path integral methods and applications, http://arXiv.org/abs/quant$\mathrm{ph} / 0004090$

Madelung, E. (1927). Quantumtheorie in hydrodynamische form. Zts. f. Phys., Vol. 40, $322-$ 326

Nairz, O., Arndt, M. \& Zeilinger, A. (2003). Quantum interference experiments with large molecules. Am. J. Phys., Vol. 71(No. 4), 319-325

Nikolić, H. (2007). Quantum mechanics: Myths and facts, Found. Phys., Vol. 37(No. 11), 15631611

Poirier, B. (2008). On flux continuity and probability conservation in complexified Bohmian mechanics. http://arXiv.org/abs/0803.0193

Poluyan, P. V. (2005). Nonclassical ontology and nonclassical movement. Kvantovaya Magiya, Vol. 2(No. 3), 3119--3134:

Sanz, A. S. \& Miret-Artês, S. (2007). A causal look into the quantum Talbot effect, J. Chem. Phys., Vol. 126, 234106

Sanz, A. S. \& Miret-Artês, S. (2008). A trajectory-based understanding of quantum interference, J. Phys. A: Math. Gen., Vol. 41, 435303

Sbitnev, V. I. (2009a). Bohmian trajectories and the Path Integral Paradigm. Complexified Lagrangian Mechanics. Int. J. Bifurcation \& Chaos, Vol. 19(No. 9), 2335-2346

Sbitnev, V. I. (2009b). N-slit interference: fractals in near-field region, Bohmian trajectories. http://arxiv.org/abs/0907.4638

Sbitnev, V. I. (2010). Matter waves in the Talbot-Lau interferometry, http://arxiv.org/abs/1005.0890

Schrödinger, E. (1926). An undulatory theory of the mechanics of atoms and molecules, Phys. Rev., Vol. 28(No. 6), 1049-1070

Stanford Encyclopeia of Philosophy, (2004). Newton's Views on Space, Time, and Motion, http:/ / plato.stanford.edu/entries/newton-stm/ 
Stanford Encyclopeia of Philosophy, (2008). Aristotle, http:// plato.stanford.edu/entries/aristotle/

Stanford Encyclopeia of Philosophy, (2010). Democritus, http://plato.stanford.edu/entries/democritus/

Struyve, W. \& Valentini, A. (2009). De Broglie-Bohm guidance equations for arbitrary Hamiltonians. J. Phys. A: Math. Theor., Vol. 42, 035301

Talbot, H. F. (1836). Facts relating to optical science. Philos. Mag., (No. 9), $401-407$

Titchmarsh, E. C. (1976). The Theory of Functions, Oxford Science Publ., Oxford

Ventzel, A. D. (1975). The course of stochastic processes theory, Nauka, Moskow, in Russian

Wyatt, R. E. (2005). Quantum Dynamics with Trajectories: Introduction to Quantum Hydrodynamics, Springer, N. Y.

Wyatt, R. E. \& Bittner, E. R. (2003). Quantum mechanics with trajectories: Quantum trajectories and adaptive grids. http://arXiv.org/abs/quant-ph/0302088

Yanov, I. \& Leszczynski, J. (2004). Computer simulation of fullerenes and fullerites. In: Computional materials science, pp. 85--115. Elsevier B. V., Netherlands 


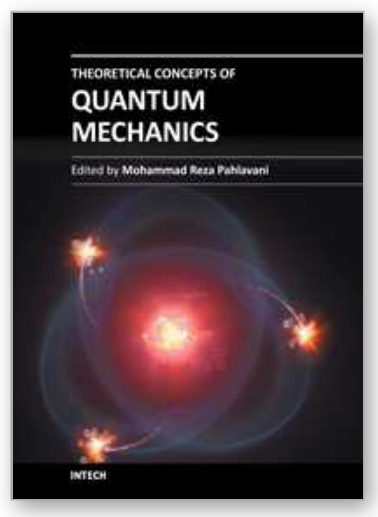

\author{
Theoretical Concepts of Quantum Mechanics \\ Edited by Prof. Mohammad Reza Pahlavani
}

ISBN 978-953-51-0088-1

Hard cover, 598 pages

Publisher InTech

Published online 24, February, 2012

Published in print edition February, 2012

Quantum theory as a scientific revolution profoundly influenced human thought about the universe and governed forces of nature. Perhaps the historical development of quantum mechanics mimics the history of human scientific struggles from their beginning. This book, which brought together an international community of invited authors, represents a rich account of foundation, scientific history of quantum mechanics, relativistic quantum mechanics and field theory, and different methods to solve the Schrodinger equation. We wish for this collected volume to become an important reference for students and researchers.

\title{
How to reference
}

In order to correctly reference this scholarly work, feel free to copy and paste the following:

Valery I. Sbitnev (2012). Bohmian Trajectories and the Path Integral Paradigm - Complexified Lagrangian Mechanics, Theoretical Concepts of Quantum Mechanics, Prof. Mohammad Reza Pahlavani (Ed.), ISBN: 978953-51-0088-1, InTech, Available from: http://www.intechopen.com/books/theoretical-concepts-of-quantummechanics/the-path-integral-paradigm-and-bohmian-trajectories-from-the-lagrangian-mechanics-tocomplexified-on

\section{INTECH}

open science | open minds

\section{InTech Europe}

University Campus STeP Ri Slavka Krautzeka 83/A 51000 Rijeka, Croatia Phone: +385 (51) 770447

Fax: +385 (51) 686166 www.intechopen.com

\section{InTech China}

Unit 405, Office Block, Hotel Equatorial Shanghai No.65, Yan An Road (West), Shanghai, 200040, China 中国上海市延安西路65号上海国际贵都大饭店办公楼405单元 Phone: +86-21-62489820

Fax: $+86-21-62489821$ 
(C) 2012 The Author(s). Licensee IntechOpen. This is an open access article distributed under the terms of the Creative Commons Attribution 3.0 License, which permits unrestricted use, distribution, and reproduction in any medium, provided the original work is properly cited. 